\title{
Erratum: Microwave Detection of Electron-Phonon Interactions in a Cavity-Coupled Double Quantum Dot [Phys. Rev. Lett. 120, 097701 (2018)]
}

T. R. Hartke, Y.-Y. Liu, M. J. Gullans, and J. R. Petta

(Q) (Received 1 October 2018; published 26 October 2018)

DOI: 10.1103/PhysRevLett.121.179901

Equations (2) and (3) contain misprints, they should be replaced by

$$
\begin{gathered}
J(\nu)=\frac{2 J_{0} c_{n}}{d \nu} \sin ^{2}\left(\nu d / 2 c_{n}\right) e^{-\nu^{2} / 2 \omega_{0}^{2}}+J_{b}(\nu), \\
J_{b}(\nu)=r J_{0} \frac{\nu}{\omega_{0}}\left[1-\operatorname{sinc}\left(\nu d / c_{n}\right)\right] e^{-\nu^{2} / 2 \omega_{0}^{2}}
\end{gathered}
$$

Neither the analysis nor the conclusions in the Letter are affected by this change. 